\title{
Implementation of Palm Print Biometric Identification System Using Ordinal Measures
}

\author{
V.K. Narendira Kumar ${ }^{1}$, Dr. B. Srinivasan ${ }^{2}$ \\ ${ }^{1}$ Assistant Profes sor, Department of Information Technology, \\ ${ }^{2}$ Associate Professor, PG\& Research Department of Computer Science, \\ Gobi Arts \& Science College (Autonomous), Gobichettipalayam-638453, Erode District, Tamil Nadu, India \\ E-mail:kumarmcagobi@yahoo.com ${ }^{1}$,srinivasan_gasc@yahoo.com ${ }^{2}$
}

\begin{abstract}
Personal identification is one of the most important require ments in alle-commerce and criminal detection applications. In this framework, a novel palm print representation method, namely orthogonal line ordinal features, is proposed. The palm print registration, feature extraction, palm print verification and palm print recognition modules are designed to manage the palm prints and the palm print database module is designed to store their palm prints and the person details in the database. The feature extraction module is proposed to extract the ordinal measurements for the palm prints. The verification module is designed to verify the palm print with the personal identification record. The recognition module is proposed to find out the relevant person associated with the palm print image. The proposed palm print recognition scheme uses the intensity and brightness to measure the ordinal measurement. The ordinal measures are estimated for the $4 \times 4$ regions of the palm print images.
\end{abstract}

Index Terms - Biometrics, Palm Print, Recognition, Ordinal Measures, Feature Extraction

\section{Introduction}

Several identification and verification schemes that exist today but the most accurate identification is in the area of biometrics. Some examples of identifying biometric characteristics are fingerprints, hand geometry, retina and iris patterns, facial geometry, and signature and voice recognition. Biometric identification may be preferred over traditional methods (e.g. passwords, smart-cards) because its information is virtually impossible to steal. Although in some cases it may become possible to impersonate a [2].

Two interesting properties of biometric identification are:

1. The person to be identified is required to physically be present at the point of identification.
2. Identification is based on the biometric technique that does not depend on the user to remember a password or to carry a token.

There are two distinct functions for biometric devices:

1. To prove you are who say you are

2. To prove you are not who you say you are not.

Palm print recognition inherently implements many of the same matching characteristics that have allowed fingerprint recognition to be one of the most wellknown and best publicized biometrics. Both palm and finger biometrics is represented by the information presented in a friction ridge impression. This information combines ridge flow, ridge characteristics, and ridge structure of the raised portion of the epidermis. The data represented by these friction ridge impressions allows a determination that corresponding areas of friction ridge impressions either orig inated from the same source or could not have been made by the same source. Because fingerprints and palms have both uniqueness and permanence, they have been used for over a century as a trusted form of identification. However, palm recognition has been slower in becoming automated due to some restraints in computing capabilities and live-scan technologies. This paper provides a brief overview of the historical progress of and future implications for palm print biometric recognition. The personal identification system using palm prints is designed to carry out the palm print recognition and verification operations. The system uses the ordinal measurement for the recognition and verification tasks. The color intensity and brightness values are used in the system. The region based ordinal measurement estimation is used in this system [1]. The system does not consider the orientation factors for the palm print comparison.

The remaining sections are organized as follows: Brief outline of palm print recognition is presented in section 2. System methodology is presented in section 3. The system implementation like database, feature extraction, verification and recognition of palm print images are briefly explained in section 4 . The testing of 
biometric system is briefly explained in section 5 . Experimental results are given in Section 6. Finally, Section 7 describes the concluding remarks.

\section{Background of the Study}

A number of palm print recognition research have been reported in the literature and most of them address the efficiency of the feature extraction algorithms. The proposed palm print representation schemes include Eigen palms (C. Harold and M. Charles, 1943), Fisher palms (X. Wu et al., 2003), Gabor code (D. Zhang et al., 2003), Competitive Code (W. K. Kong and D. Zhang, 2004), Ordinal feature (Z. Sun et al., 2005), line features (J. Fonda et al., 1998), and feature points (D. Zhang and W. Shoo, 1999). However, not much detail of the palm print acquisition method was provided although the acquisition process is one of the key considerations in developing a fast and robust online recognition system. In earlier study, inked-based palm print images (J. Fonda et al., 1998) (D. Zhang and W. Shoo, 1999) were used. The palm prints were inked to paper and digitized using scanner. The two-step process was slow and is not suitable for online system. Recently, various input sensor technology like flatbed scanner, CCD camera, CMOS camera, and infrared sensor have been introduced for more straight-forward palm print acquisition. Among the technology, scanner and CCD camera are the commonly used input devices (C. Harold and M. Charles, 1943) (X. Wu et al., 2003). Scanner and CCD camera are able to provide very high quality images with little loss of information. However, the process of scanning a palm image requires some time (a few seconds) and the delay cannot cope with the requirement of an online system. Zhang et al. (D. Zhang et al., 2003) proposed the use of CCD camera in semi-closed environment for online palm print acquisition and good results had been reported by using this approach. In this paper, we explore the use of a low-resolution web-cam for palm print acquisition and recognition in real-time system.

\subsection{Objectives of the System}

The person identification system is designed to perform the person identification operation using the palm prints. The palm print recognition and verification are the main objectives of the system. The ordinal measures can be used to rank and order the palm print images. The system should perform the recognition and verification operation orientation independently. The region based comparison technique can be applied for the system. The ordinal feature and edge feature are the main features for the palm print recognition process. The orientation independent personal identification process is the main motive for the system. The person identification with palm print recognition and verification is the design goal of the system.

\subsection{Approach}

Palm identification, just like fingerprint identification, is based on the aggregate of information presented in a friction ridge impression. This information includes the flow of the friction ridges the presence or absence of features along the individual friction ridge paths and their sequences and the intricate detail of a single ridge. To understand this recognition concept, one must first understand the physiology of the ridges and valleys of a fingerprint or palm. When recorded, a fingerprint or palm print appears as a series of dark lines and represents the high, peaking portion of the friction ridged skin while the valley between these ridges appears as a white space and is low, shallow portion of the friction ridged skin.

Palm recognition technology exploits some of these palm features. Friction ridges do not always flow continuously throughout a pattern and often result in specific characteristics such as ending ridges or dividing ridges and dots. A palm recognition system is designed to interpret the flow of the overall ridges to assign a classification and then extract the minutiae detail a subset of the total amount of information available, yet enough information to effectively search a large repository of palm prints. Minutiae are limited to the location, direction and orientation of the ridge endings and bifurcations' (splits) along a ridge path. A pictorial representation of the regions of the palm, two types of minutiae, and examples of other detailed characteristics used during the automatic classification and minutiae extraction processes.

\section{System Methodol ogy}

Biometrics makes use of the physiological or behavioral characteristics of people such as fingerprint, iris, face, palm print, gait, and voice, for personal identification, which provides advantages over nonbiometric methods such as password, Personal Identification Number, and ID cards [3].

Palm print is the unique inner surface pattern of human hand, including a number of discriminating features, such as principal lines, wrinkles, ridges, minutiae points, singular points, texture etc. Compared with other bio metric traits, the advantages of palm print are the availability of large palm area for feature extraction, the simplicity of data collection and high user acceptability. Various palm print representations have been proposed for recognition such as Line features, Feature points, Fourier spectrum, Eigen palms features, Sobel's and morphological features, Texture energy, Wavelet signatures, Gabor phase, Fusion code, Competitive code, etc.

The Iris biometric scheme is attempt to explore a convincing solution, that of using ordinal measures, as an answer to the representation problem. The representation of ordinal measures unifies several state- 
of-the-art palm print recognition algorithms of David Zhang. The internal representations of those algorithms can be seen as special cases of ordinal measures. This form a framework, which may help to understand the discriminate power of palm print pattern, guide further research and enlighten new ideas (see figure 1).

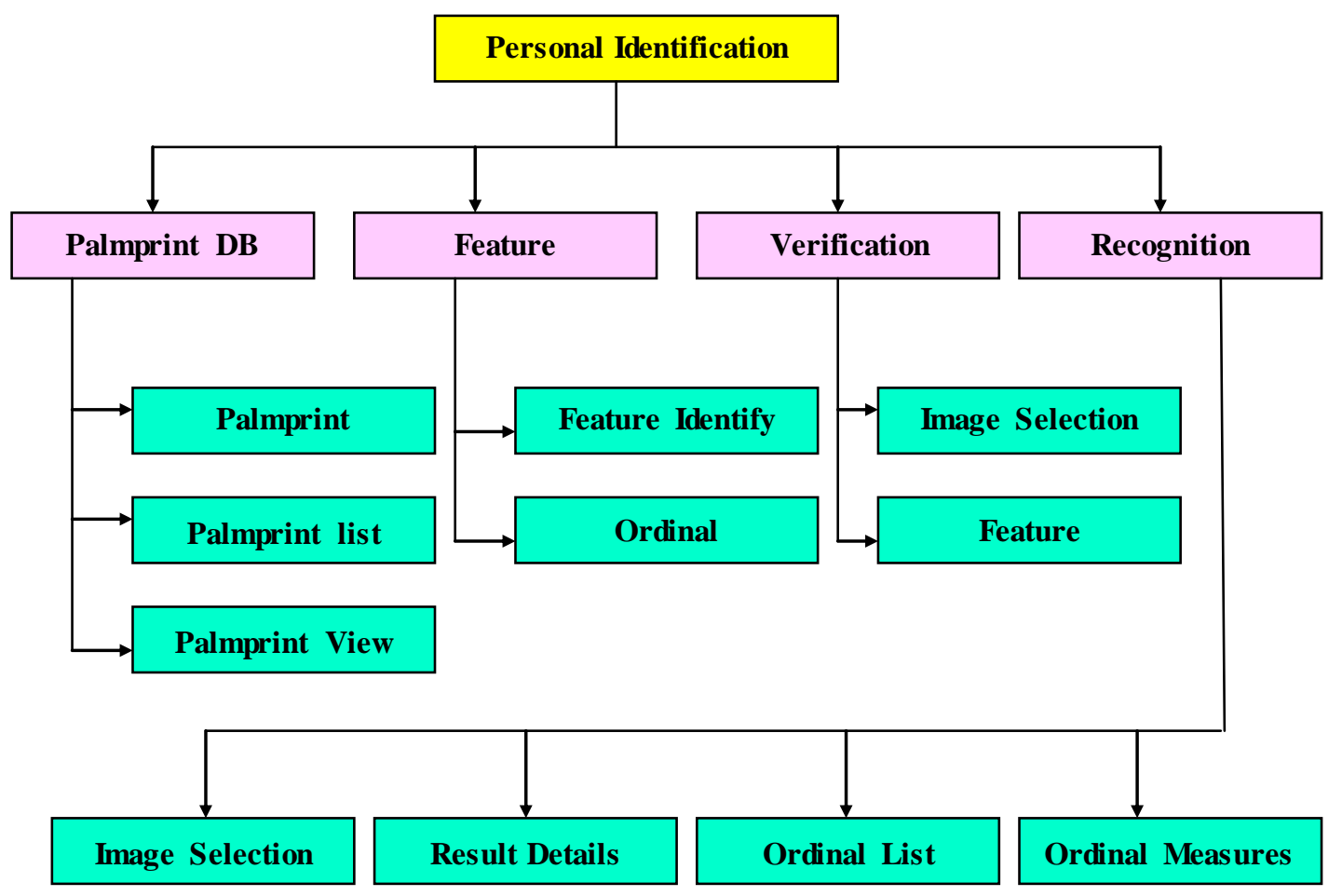

Fig. 1: Palm Print System Architecture

\subsection{Ordinal Measures}

Ordinal measures come from a simple and straightforward concept. For example, system could easily rank or order the heights or weights of two persons, but it is hard to answer their precise differences [7]. For computer vision, the absolute intensity information associated with an object can vary because it can change under various illumination settings. However, ordinal relationships among neighborhood image pixels or regions present some stability with such changes and reflect the intrinsic natures of the object. The symbols denote the inequality between the average intensities of two image regions. The inequality represents an ordinal relationship between two regions and this yields a symbolic representation of the relations. For digital encoding of the ordinal re lationship, only a single bit is used. For example, " 1 " denotes " $\mathrm{A}>\mathrm{B}$ " and " 0 " denotes " $\mathrm{A}<\mathrm{B}$ " and the equality case can be assigned to either.

\subsection{Unified Framework for Palm Print Recognition}

A general framework of palm print recognition is based on the ordinal representation is proposed. To input a palm print image, the central sub image in the aligned coordinate system is cropped from it for feature extraction. To obtain the special measurements for ordinal comparison, the normalized palm image is transformed to feature image. Then the ordinal measures are obtained by qualitatively comparing several quantities in feature image. In practice, the transformation and ordinal comparison can be combined into one step via differential filtering. The result of ordinal comparison may be the sign of an inequality, the rank order of all measurements involved in comparis on, maximu m or minimu m value as sociated inde $x$ and so on. After ordinal comparison, all results are coarsely quantized into binary bits so as to strengthen the robustness of palm feature and facilitate matching step. All binary codes are concatenated to generate a palm print feature, which is the input of matching engine. Finally, the dissimilarity between the input palm print's ordinal feature and the template stored in database is measured by their Hamming distance.

The framework has some desirable properties for palm print recognition: The ord inal measure renders the palm print representation robust against various intra-class variations such as illumination settings, dirties or sweats on palm, signal noises, pose change, misalignment and nonlinear deformations [4].

Each bit palm print feature code represents an ordinal relationship among several image regions, which is rich of information. Because the palm print code has equal probability to be 1 or 0 for an arbitrary 
pattern, its entropy is maximized. Although the discriminability of a single palm code is limited, a composite palm template formed by numerous of ordinal feature codes has sufficiently high degrees-offreedom to differentiate all individuals in the world. Thus the randomness of palm print pattern is well encoded. The dissimilarity between two palm prints can be measured by bitwise XOR operator, which could be computed on-the-fly [8].

\subsection{Gabor Based Representations}

Based on the proposed framework, the system the Gabor based representations proposed by Zhang, which reported the best recognition performance in literature, are special cases of ordinal measures. Gabor based encoding filters used in palm code are essentially ordinal operators. For odd Gabor filtering of local palm print region, the image regions covered by two excitatory lobes are compared with the image regions covered by two inhibitory lobes. The filtered result is qualitatively encoded as " 1 " or " 0 " based on the sign of this inequality. Similarly, even Gabor generated palm code is mainly determined by the ordinal relationship between one excitatory lobe-covered region and two small inhibitory lobes-covered regions. Because the sum of orig inal even Gabor filter's coefficients is not equal to 0 , the average coefficient value is reduced from the filter to maximize the information content of the corresponding palm code.

However, ordinal relationship is not restricted to intensity measurement. As a byproduct of Gabor phase measure the orientation energy or magnitude was also obtained by orthogonal Gabor filtering. Thus it is possible to combine ordinal intensity measures and ordinal energy measures together. In, the local energy along four different orientations were compared each other to obtain the maximum. Then the palm print is represented using the Gabor filtered ordinal intensity measures whose basic lobes are along the maximum energy orientation. Fusion code was demonstrated as a more discriminative representation than single type ordinal measure based representation. (a) Odd Gabor filter (orientation $=45^{\circ}$ ). (b) Ordinal comparison of image regions using odd Gabor filter, "+" denotes excitatory lobe covered image region and "." represents inhibitory lobe covered image region. Even Gabor filter (orientation $=45^{\circ}$ ). (d) Ordinal comparis on of image regions using even Gabor filter.

The field of palm print recognition, competitive code proposed by Kong performs the best in terms of accuracy. There, each palm print image region has a dominant line segment and its orientation is regarded as the palm print feature. Because the even Gabor filter is well suited to model the line segment, it was used to filter the local image region along six different orientations, obtaining the corresponding contrast magnitudes. Based on the winner-take-all competitive rule, the index of the minimum contrast magnitude was represented by three bits, namely competitive code. The success of this method also depends on ordinal measures because the process of competition for winner is based on ordinal comparis on essentially. The ordinal measures, these algorithms all perform well in large scale testing, in terms of both accuracy and efficiency. Therefore, the ordinal measures are perhaps the most suitable representation for palm print-based identification system.

\subsection{Orthogonal Line Ordinal Features}

The ultimate purpose of the proposed framework is to guide the development of new algorith ms. Following the framework, a possible improvement could be made by choosing well-designed ordinal measures as the palm print representation, into which the characteristics of palm print pattern should be incorporated. A novel palm print representation, namely is proposed, Orthogonal Line Ordinal Features (OLOF), where normalized sub image is referenced by finger gaps using an algorithm similar to Zhang. OLOF is so called because the two regions involved in ordinal comparis on are elongated or line-like, and the two are geometrically orthogonal.
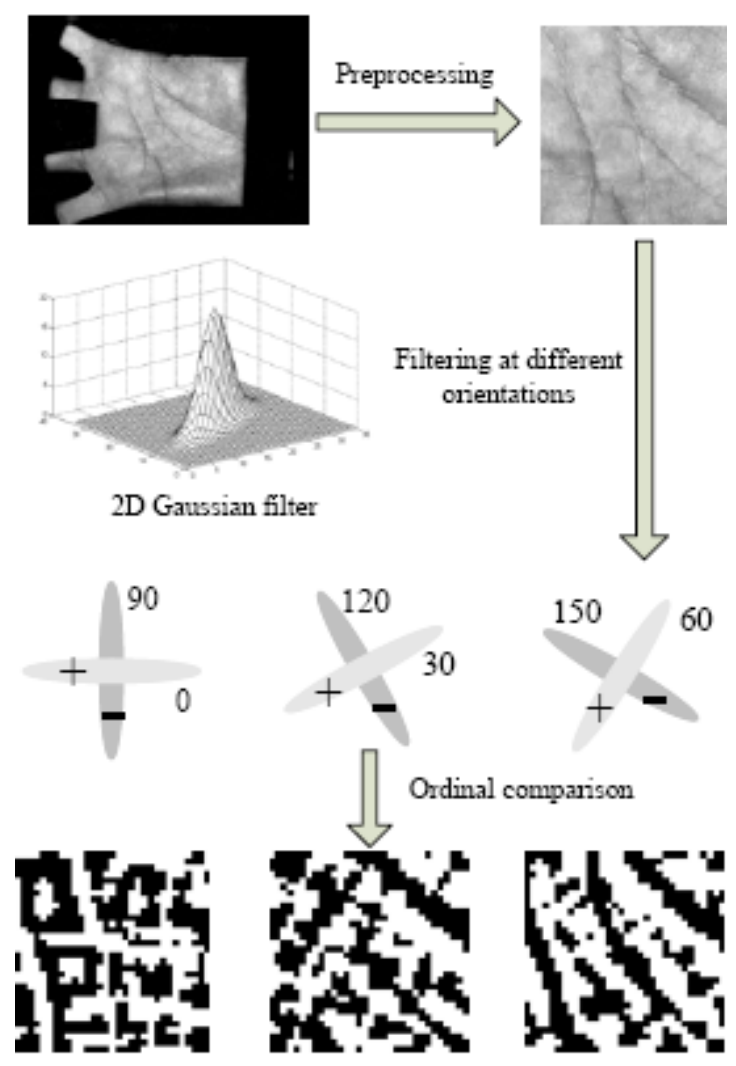

Ordinal comparison

Ordinal code

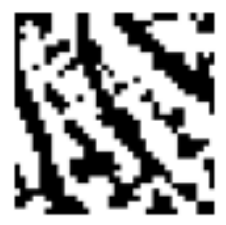

Fig. 2: Orthogonal line ordinal features for palm print recognition

The ideas are motivated by the most stable and robust ordinal measures available in palm print pattern, i.e. randomly distributed negative line segments versus their orthogonal regions. In low-resolution palm print 
images, the line patterns are mainly constituted by principal lines and wrinkles, whose intensity is much lower than their orthogonal regions. Of course detection of all line segments in palm print is impossible in real time applications. Nevertheless if system applies thousands of ordinal operators onto a palm print image, most of them correspond to robust ordinal measures. This assumption is verified in the following experiments. 2D Gaussian filter to obtain is used the weighted average intensity of a line-like region. Its expression is as follows:

$$
\begin{aligned}
f(x, y, \theta) & =\left[\exp \left[-(x \cos \theta+y \sin \theta)^{2} / \delta x\right]\right. \\
& \left.-\left[(-x \sin \theta+y \cos \theta)^{2}\right] / \delta y\right]
\end{aligned}
$$

Where $\varphi$ denotes the orientation of 2D Gaussian filter denotes the filter's horizontal scale and $\delta_{y}$ denotes the filter's vertical scale. Control the scale ratio $\delta_{\mathrm{x}} / \delta_{\mathrm{y}}$ higher than 3 to make its shape like a line. The orthogonal line ordinal filter, comparing two orthogonal line-like palm print image regions, is specially designed as follows:

$$
\text { OF }(\theta)=f(x, y, \theta)-f(x, y, \theta+\Pi / 2)
$$

For each local region in normalized palm print image, three ordinal filters, $\mathrm{OF}(0), \mathrm{OF}(\pi / 6)$ and $\mathrm{OF}(\pi$ / 3), are performed on it to obtain three bit ordinal codes based on the sign of filtering results. Finally, three ordinal templates named as ordinal code are obtained as the feature of the input palm print image see figure 2. The matching metric is also based on Hamming distance.

\section{Palm Print System Implementation}

The palm print verification and recognition system is implemented to perform the person identification operations. The palm prints are one of the important biometric features that are used to identify a person with unique properties. The palm print and relevant person details are maintained in the database. Palm print features such as line, edge, ridge and orientation features are maintained in the database. This system uses a new feature named as dominal features. The dominal features are extracted with reference to the color and texture properties. The person identification task is divided into two types. They are biometric verification and recognition operations. The palm print is used as the biometric features for the person. The verification process performs the similarity checking for the given person id and the palm print image. The palm print database image for the specified person is compared with the input image. The verification process requires only one comparison process. The recognition is the process of identifying an unknown person with reference to the biometric features. In this mechanis $m$ the palm print database images are compared with the given input image. Most relevant image is referred as similar palm print for the person.

The person identification using palm prints is developed as a graphical user interface based application. The Java language is used as the front end and the Oracle relational database is used as the back end for the system. The system is divided into four major modules. They are palm print database, feature extraction, verification and recognition. The palm print database module is designed to manage the palm print and person details under the database. The feature extraction module is designed to fetch the ordinal and line features for the palm prints. The palm print verification for the given person is performed in the verification module.

\subsection{System Development}

The personal identification using palm print system is developed as a graphical user interface based tool. The system is developed using the J2EE language and the Oracle relational database. Palm print features and person details are maintained in the database. The palm print images are maintained with their id. A separate folder is allocated to maintain the palm print images.

An online system captures palm print images using a palm print capture sensor that is directly connected to a computer for real-time processing. The palm print images are captured in two ways. They are image scanners and thermal scanners. The thermal scanners are used to capture the palm print images directly from the hand. The image scanners are used capture the palm print images from pictures. The images are maintained in two formats. They are Joint Photographical Expert Group (JPEG) and Graphical Interchange Format (GIF). The palm print images are divided into $4 \times 4$ regions. The 16 regions are used for the feature extraction process. The color brightness and intensity values are used for the feature extraction process. The feature values are maintained for each image under the database. The verification process is done with the person id and the palm print image. The recognition process is carried out with the palm print image only. The verification and recognition operations are conducted with a threshold value for the similarity rate estimation process.

\subsection{Palm Print Database}

The palm print database module is the initial module in this application. The personal detail and palm print data are maintained in the database. Palm prints are maintained as image files. The palm print database updates the palm print image name under the database. The palm print database also maintains the color and ordinal features in the database. The ordinal values for 16 regions are maintained in the database. The system also maintains the rank or order for the palm prints 
with reference to its ordinal values. The ranking values are used in the recognition process. The person details and ordinal features are maintained in two different tables [5].

The palm print database module is divided into three sub modules. They are palm print registration; palm print list and palm print view. The palm print registration sub module is designed to register a person details with the palm print information. The person name, address, city, state, country and e-mail details collected and updated into the database. The system assigns a unique person identification number for the registered persons. The palm print image path is also registered into the palm print registration process [9]. The palm print is copied into the images folder maintained in the application path. The palm print image is renamed with reference to the person identification number assigned by the system.

The file open dialog window option supports the palm print path selection process. The user can easily select the path for the palm print image. The palm print list sub module shows the list of registered person details with the palm print details. The palm print list is prepared by using the palm print database. The palm print image view sub module is designed to print the palm print for the selected person from the palm print list.

\subsection{Feature Extraction}

The feature extraction module is designed to extract and update the palm print image features. The recently updated palm print image features are extracted and updated under the database. The color and texture feature are the main feature that considered in this system. The color and texture feature is referred as ordinal features. The line features are used for the comparis on process. The palm print image pixel values are used for the feature extraction process. The color brightness and intensity values are used for the feature extraction process.

The feature extraction module is divided into two sub modules, feature identification and ordinal measurement. The feature identification process is carried out over the regions on the palm print images. The palm print image is divided into $4 \times 4$ regions. All the 16 image regions are passed into the feature extraction process. The color intensity and brightness for each block is compared with another palm print image. The ordinal values are estimated with reference to the color brightness and intensity values. The ordinal values are represented as 0 's and 1's. The block ordinal value is summed and they are compared with the similar block in the other palm print image. The comparis on results are also maintained as 0's and 1's. The total ordinal measure for the image is calculated and assigned for the image. The ordinal measurement sub module displays all the ordinal measurements and ratio level for each image.

\subsection{Palm Print Verification}

The palm print verification module is designed to carry out the person's identification tasks. The verification process performs the palm print comparison process only one iteration [1]. The person id and input palm print image are collected from the user. The palm print selection module is designed for the palm print selection process. The feature for the input palm print is extracted and compared with the palm print image features that are maintained for the relevant person under the database. The feature comparison module is designed to carry out the comparison process [6]. The ordinal features and line features are used for the feature comparison process. The false acceptance ratio and false rejection ratio are used for the comparison process. The final result of the verification process is given as the palm print which is similar to the referred person.

\subsection{Palm Print Recognition}

The palm print recognition module is designed to carry out the person identification process for the unknown person. The palm print image is the only input data for the recognition process. The person identification details are the expected output value. The input image feature is compared with the database image features. The relevancy is estimated with reference to the threshold value. The most relevant image is selected for the person's identification. If the comparison result does not match with the input image then the recognition process is declared as unknown person.

The recognition module is divided into four sub modules. They are palm print selection, result details, ordinal list and ordinal measurement. The palm print image selection sub module is designed to select the palm print input image. The file open dialog is used to select the input image file. The result details produce the list of relevant palm print with their similarity ratio details. The ord inal list shows the ordinal feature based comparisons. The ordinal measurement sub module shows the ordinal values for each region.

\section{Testing of Biometric System}

Testability is the "degree to which a system or component facilitates the establishment of test criteria and the performance of tests to determine whether those criteria have been met". Testability is a concern in both hardware and software designs. In hardware design, testability implies that there exist tests to detect any fault with respect to a fault model in a finished product. Thus, the aim is to verify the correctness of a fin ished product. Testability in software focuses on the verification of design and implementation. Testing is expensive in terms of money, time, and resources. On the other hand, the test must be rigorous enough to 
yield a very close approximation of the inherent matching capabilities of the biometric system. This test plan was proposed for a former client. The objective of the test was twofold; determine the inherent accuracy of the system, and, develop a set of "samples" a "test set" that could be used in future accuracy tests of the systemas the systems database(s) grew in size.

\subsection{Palm Print Liveness Testing}

Biometrics can be fooled by presenting an artificial or simulated palm print biometric sample. What is required for protecting against these attacks, is liveness testing: an automated test performed to determine if the sample presented to the system comes from a live human being. Consider Examples for palm print [7]:

A palm print scanner by Sony FIU-500 is an optical sensor. However, it tests for liveness by measuring the capacitance of the skin. If it is not within norms, the sample is rejected. A method developed at West Virginia University captures the perspiration pattern using a capacitive palm print scanner. Live palm sweats, pores fill with moisture, and the capacitance changes. The measurement is done in 5 seconds. Other things than capacitance can be measured: Properties of a living body: Mechanical: weight, density, elasticity. Electrical: resistance, impedance, dielectric constant. Visual: color, opacity, appearance. Signals generated by a living body: Pulse, blood pressure, heat, thermal gradients, electrical signals generated by the heart, etc. Responses to a stimulus: Voluntary: Tactile, visual. Auditory: respond to feeling/seeing/hearing something. Involuntary: Electro myography, pupil dilation, reflexes.

\section{Experimental Results}

Palm print images from 284 individuals using the palm print capture device as described in are collected. In this dataset, 186 people are male, and the age distribution of the subjects is: about $89 \%$ are younger than 30 , about $10 \%$ are aged between 30 and 50, and about $1 \%$ is older than 50 . The palm print images were collected on two separate occasions, at an interval of around two months. On each occasion, the subject was asked to provide about 10 images each of the left palm and the right palm. Therefore, each person provided around 40 images, resulting in a total number of 11,074 images from 568 different palms in our database. The average time interval between the first and second occasions was 73 days. The maximum and the minimum time intervals were 340 days and 1 day, respectively. The size of all the test images used in the following experiments was 384'284 with a resolution of 75dpi. The database is divided into two datasets, training and testing. Testing set contained 9,599 palm print images from 488 different palms and training set contained the rest of them.
The training set is used to adjust the parameters of the Gabor filters only. All the experiments were conducted on the testing set see figure 3, 4, 5, and figure 6 . We should emphasize that matching palm prints from the same sessions was not counted in the following experiments. In other words, the palm prints from the first session were only matched with the palm prints from the second session. A matching is counted as a genuine matching if two palm print images are from the same palm; otherwise it is counted as an imposter matching. Number of genuine and imposter matching are 47,276 and 22,987,462 respectively.

A feature-level coding scheme for palm print identification is presented. On the top of Palm Code, a number of improvements for developing Fusion Code are made. 1) The circular Gabor filter in Palm Code is replaced by a bank of elliptical Gabor filters. 2) A feature level fusion scheme is proposed to select a filter output for feature coding. 3) The static threshold in Palm Code is replaced by the dynamic threshold. A series of experiments has been conducted to verify the usefulness of each improvement.

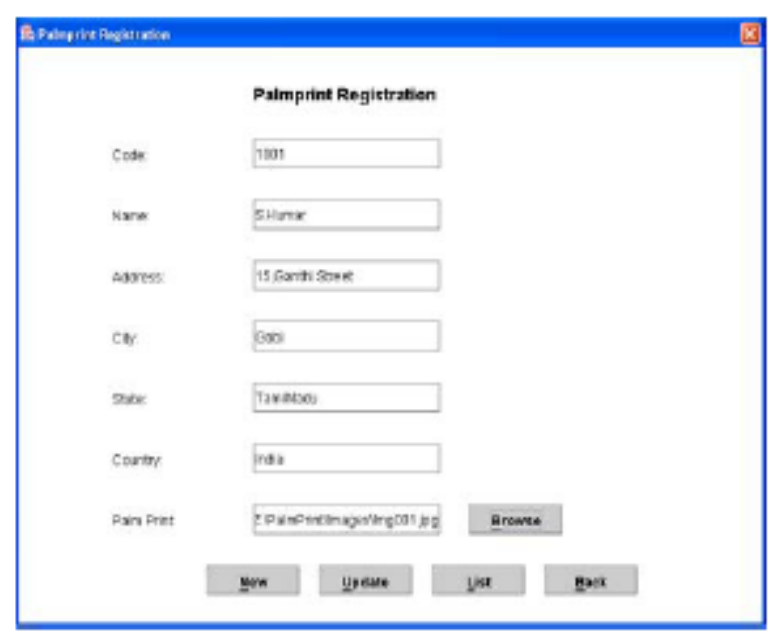

Fig. 3: Output Form 1: Palm Print Registration

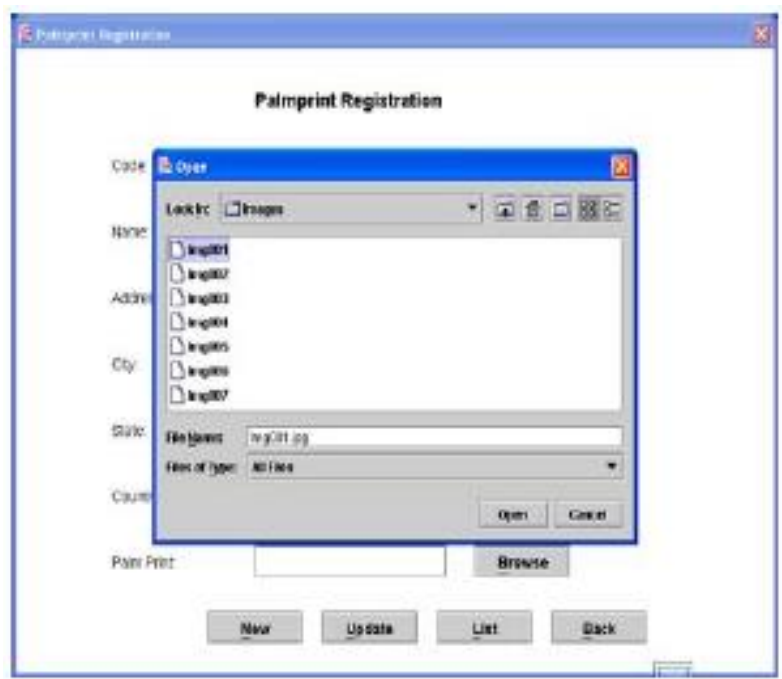

Fig. 4: Output Form 2: Palm print Registration 


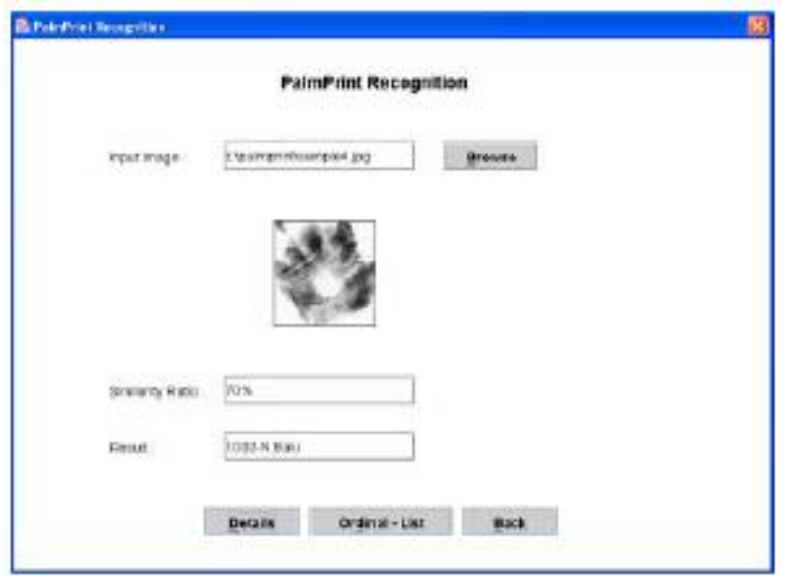

Fig. 5: Output Form 4: Palm Print Recognition

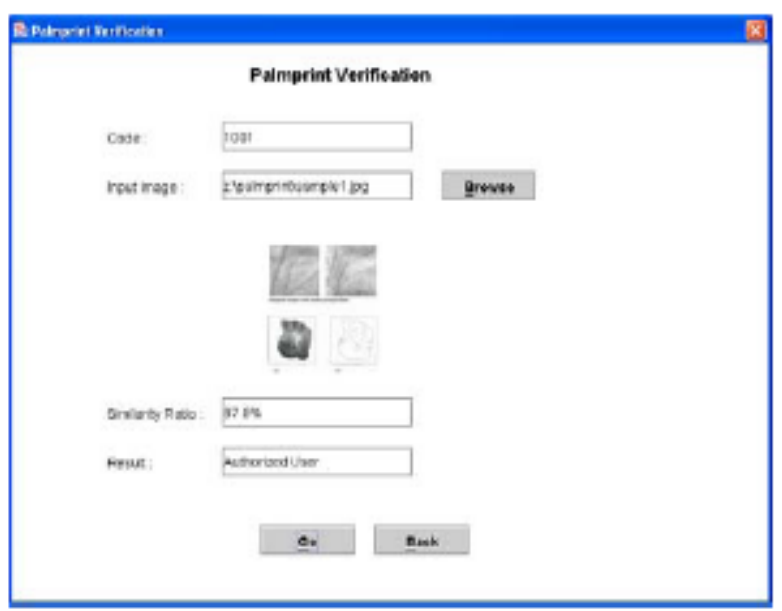

Fig. 6: Output Form 5: Palm Print Verification

Ordinal measures are robust against illumination, contrast and misalign ment variations. The experimental results proved that ordinal measures are the most suitable for palm print feature representation. On the other hand, the results also proved the statistical richness of ordinal information in palm print. Although these palm codes are all based on ordinal measures, their recognition performance could differ to a large extent due to the use of different ord inal operators. In terms of accuracy, ordinal code performs the best, followed by competitive code, fusion code and palm code. Another compelling advantage of our ordinal code is that its processing speed is nearly twice fast as that of competitive code.

\subsection{Performance of Palm Print Biometrics}

In this experiment, different numbers of elliptical and circular Gabor filters are examined. For the circular Gabor filters, I use the previous parameters for these comparisons. Figure 7(a) shows the four ROC curves obtained from elliptical Gabor filters. Each of the ROC curve represents different numbers of Gabor filters used in the fusion rule. Figure 7(b) shows the results obtained from the circular Gabor filters. In this test, the static threshold is used, rather than the dynamic threshold. According to Figure 7, we have two observations. 1) The elliptical Gabor filters perform better than the circular Gabor filters. 2) Using two filters for fusion is the best choice for both cases. The first observation can be easily understood. The elliptical Gabor filters have more parameters so that they can be well tuned for palm print features. The reason for the second observation is not obvious. Therefore, another set of experiments is conducted.

In this set of experiments, the elliptical case is considered only. First of all, the imposter distributions without considering translated matching are plotted in Figure 8(a). We can see that the imposter distributions from two to four filters are very similar. Their means, $\mu_{s}$ are 0.497 and standard deviations, $\sigma_{s}$ are around 0.0258 . However, the imposter distribution from a single filter has a relatively large variance. If bino mial distribution is used to model the imposter distributions, the imposter distributions from two to four filters have around 370 degrees-of-freedom. Ho wever, the imposter distribution from the single filter only has 250 degrees of-freedom. The degrees-of-freedoms are estimated by $\mu_{s}\left(1-\mu_{s}\right) / \sigma_{s}{ }^{2}$. These values demonstrate that using more than two filters cannot improve the imposter distributions but increasing number of filters from one to two can get a great improvement. Although increasing number of filters can reduce the variances of the imposter distributions, it would adversely influence the genuine distributions. Given two patches of palm prints from the same palm and same location, if the number of filters is increased, the fusion rule has high probability to select different filters for coding. To demonstrate this phenomenon, all the palm prints from the same hand is matched. If the fusion rule selects the same filter, the matching distance of these local patches is zero; otherwise it is one. Then, the local matching distances are summed as a global matching distance for comparing two palm prints. The global matching distance is normalized by the matching area. In other words, the matching function is still a hamming distance.

Figure 8(b) shows the cumulative distributions of the genuine hamming distances. We see that the fusion rule using four filters is the easiest to select different filters. When the hamming distance is shorter than 0.3 , the fusion rule using three filters performs better than that using two filters. It contradicts our expectation. The reason is that the direction of one of the three filters is close to one of the principal lines. Thus, it provides an extra robustness to the filter selection. Nevertheless, when the hamming distance is longer than 0.3 , fusion rule using two filters performs better. This range is more important since false acceptance tends to happen in that region. Combining the influences for the imposter and genuine distributions, the best choice is to employ two filters for fusion. In the following experiments, I study only the two elliptical filters case. 


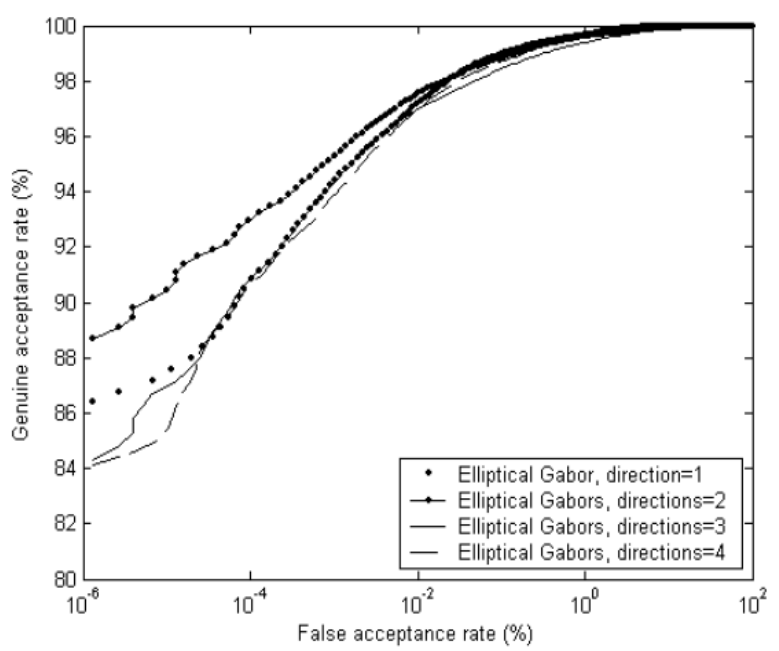

(a)

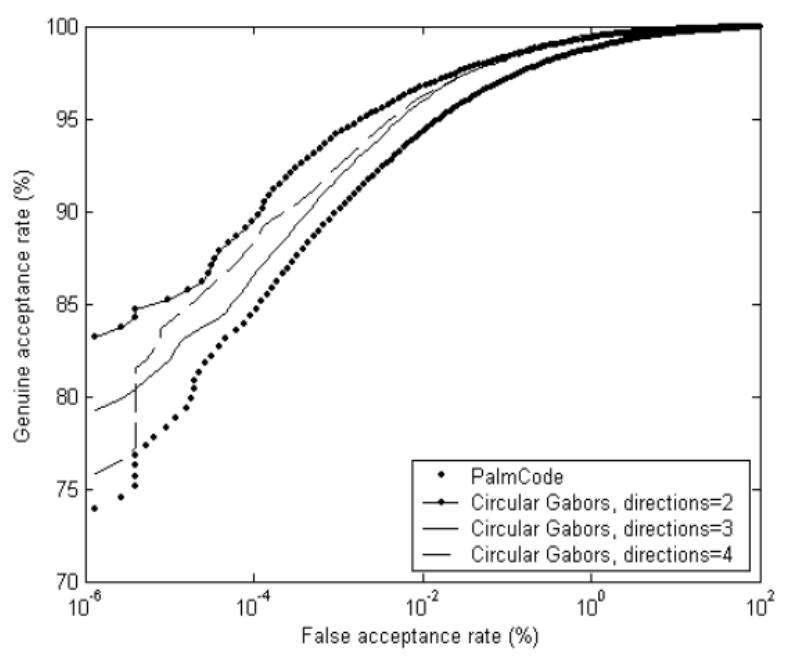

(b)

Fig. 7: Comparisons between different numbers of filters used in fusion, (a) elliptical Gabor filters and (b) circular Gabor filters

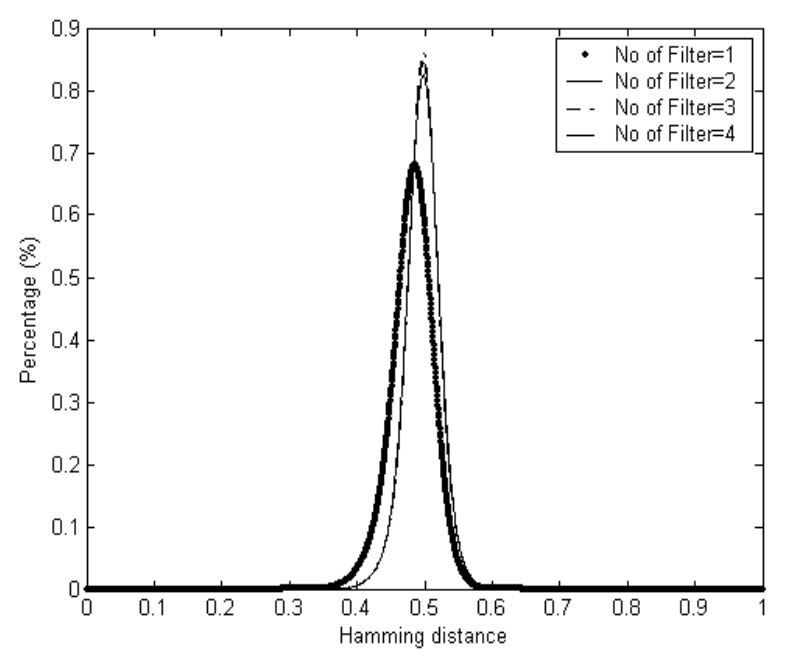

(a)

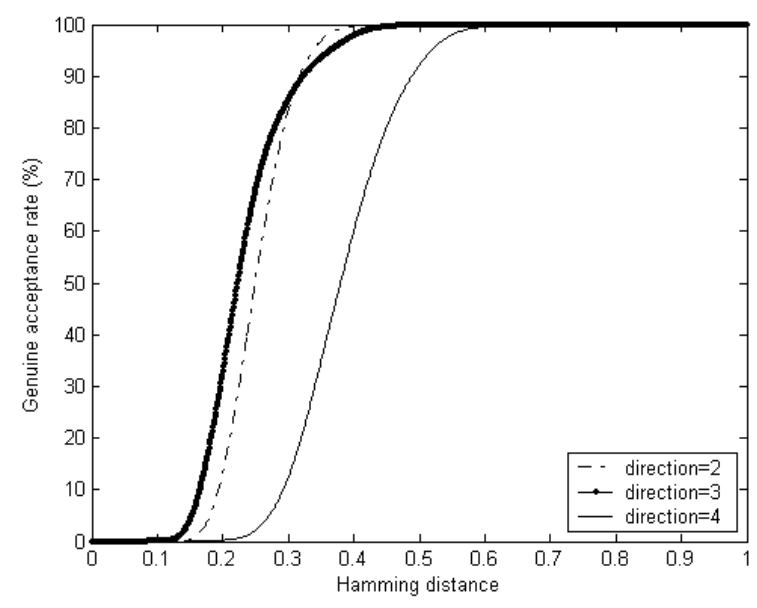

(b)

Fig. 8: Analysis of different numbers of filters for fusion. (a) Comparison between imposter distribut ions using different numbers of elliptical Gabor filters for fusion. (b) The cumulative distributions of hamming distance for studying the fusion rules selecting different filters for coding

The proposed dynamic threshold and original static threshold are compared. For graphical presentation convenience, I dynamically scale the hamming distances rather than the threshold. In fact, they have the same effect. Figure 9 shows their ROC curves. We can see that dynamic threshold effectively improves the accuracy. Combining all the proposed improvements including elliptical Gabor filters, fusion rule and dynamic threshold, the proposed method obtains around $15 \%$ improvement for genuine acceptance rate when the false acceptance rate is $10^{-6} \%$.

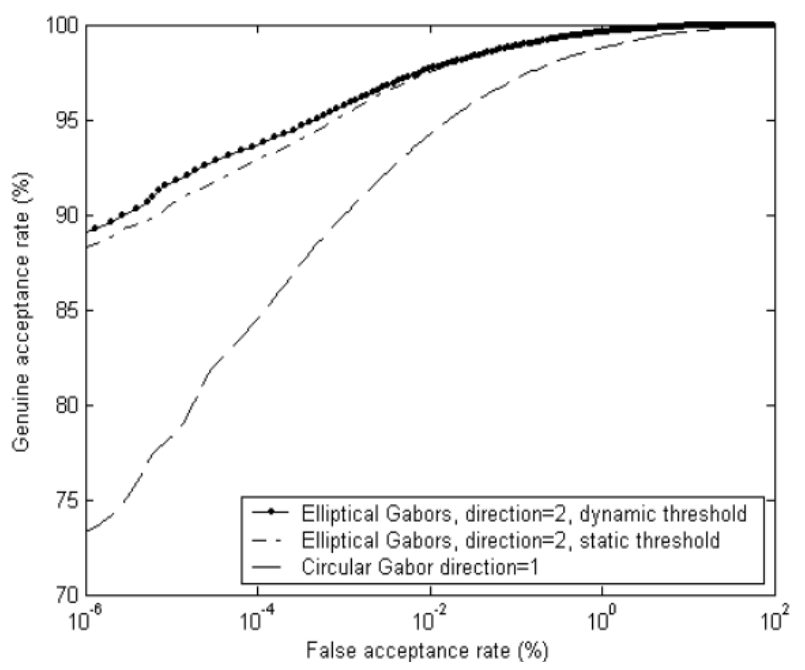

Fig. 9: Comparison between dynamic and static thresholds

Table 1(a) lists some false acceptance rates and false rejection rates and the corresponding thresholds. The results demonstrate that the proposed method is comparable with the previous palm print approaches and other hand-based biometric technologies, including hand geometry and fingerprint verification [7]. It is also comparable with other fusion approaches [5]. A 
detailed comparison between Fusion Code and other palm print algorithms can be in Competitive Coding
Scheme for Palm print Identification.

Table 1: Genuine and false acceptance rates with different threshold values, (a) Verification results and (b) 1 -to-488 identification results

(a)

\begin{tabular}{|c|c|c|}
\hline Threshold & False Acceptance Rate & False Rejection rate \\
\hline 0.317 & $1.2 \times 10^{-5} \%$ & $7.77 \%$ \\
\hline 0.324 & $1.3 \times 10^{-4} \%$ & $6.07 \%$ \\
\hline 0.334 & $1.0 \times 10^{-3} \%$ & $4.15 \%$ \\
\hline 0.350 & $1.0 \times 10^{-2} \%$ & $2.32 \%$ \\
\hline
\end{tabular}

(b)

\begin{tabular}{|c|c|c|}
\hline Threshold & False Acceptance Rate & False Rejection Rate \\
\hline 0.309 & $6.91^{\prime} 10^{-3} \%$ & $4.56 \%$ \\
\hline 0.315 & $1.38^{\prime} 10^{-2} \%$ & $3.67 \%$ \\
\hline 0.323 & $1.24^{\prime} 10^{-1} \%$ & $2.61 \%$ \\
\hline 0.333 & $9.68^{\prime} 10^{-1} \%$ & $1.74 \%$ \\
\hline
\end{tabular}

\section{Conclusion}

The person identification system uses the palm print biometric feature to identify a person uniquely. The palm print images applied into the transformation and ordinal measurement evaluation process. The ordinal measures are used to rank and order the palm print images. The system performs the region based comparis on mechanis $m$ to carry out the recognition and verification operations. The palm print database is updated with a set of personal details and palm print image collection. The feature extraction process is verified and the ranking process is also validated. The palm print recognition and verification operations are tested with a set of sample image collections. The ordinal measures and similarity ratio are estimated and verified for each analysis process. The similar palm prints are identified with high similarity ratio and irrelevant palm print images are not recognized by the system. The system provides the palm print recognition mechanis $m$ without the orientation related factors. The ordinal measurement based upon the person's identification mechanis $m$ is a feasible solution for the personal identification requirements.

\section{References}

[1] A.K. Jain, Ruud Bolle and Sharath Pankanti "Palm print Biometrics: Personal Identification in Networked Society" Publisher: Springer; 2nd printing edition 2005. Page No. 56-64.

[2] D.D. Zhang "Palm print Authentication" Publisher: Springer; 1st Edition 2004. Page No. 25-36.
[3] Harold L. Alexander "Classifying Palm prints: A Complete System of Coding, Filing and Searching Palm prints" Publisher: Charles C Thomas Pub Ltd 2009. Page No. 98-108.

[4] John D. Woodward Jr. and Nicholas M. Orlans "Biometrics" McGraw-Hill Osborne Media; 1st Edition 2002. Page No. 12-19.

[5] John R. Vacca "Biometric Technologies and Verification Systems" Butterworth-Heinemann; 1st edition 2007. Page No. 88-93.

[6] K.S. Thyagarajan "Palm print Image Processing with Applications", Focal Press, 2005. Page No. 256-272.

[7] Kenneth R. Castleman "Palm print Image Processing" Prentice Hall 2010. Page No. 72-77.

[8] Patricia Anne Kolb "H.I.T: A manual for the classification, filing, and retrieval of palm prints" Publisher: Thomas 2011. Page No. 122-129.

[9] Samir Nanavati, Michael Thieme and Raj Nanavati "Palm print Biometrics: Identity Verification in a Networked World" Publisher: Wiley; 1st edition 2002. Page No. 32-44.

\section{Authors' Profiles}

Mr. V.K. NARENDIRA KUMAR M.C.A., M.Phil., Assistant Professor, Department of Information Technology, Gobi Arts and Science College (Autonomous), Gobichettipalayam - 638 453, Erode District, Tamil Nadu, India. He received his M.Phil Degree in Computer Science from Bharathiar University in 2007. He has author more than 30 international journal article publications. He has 


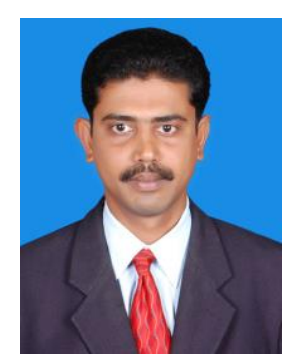

authored or co-authored more than 55 technical papers and conference presentations. He is an editorial board member for several international journals. His research interests are focused on Internet Security, Biometrics, Advanced Networking, Visual Hu man-Computer Interaction, and Multiple Biometrics Technologies.

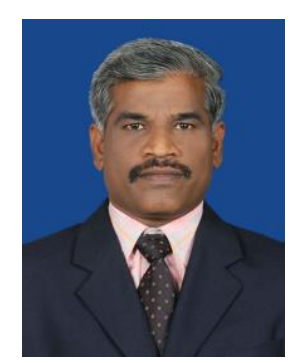

Dr. B. SRINIVASAN M.C.A., M.Phil., M.B.A., Ph.D., Associate Professor, PG and Research Department of Computer Science, Gobi Arts \& Science College (Autonomous), Gobichettipalayam - 638 453, Erode District, Tamil Nadu, India. He received his Ph.D. Degree in Computer Science from Vinayaka Missions University in 11.11.2010. He has authored or co-authored more than 70 technical papers and conference presentations. $\mathrm{He}$ is a reviewer for several scientific e-journals. His research interests include automated biometrics, computer networking, Internet security, and performance evaluation.

How to cite this paper: V.K. Narendira Kumar, B. Srinivasan,"Implementation of Palm Print Biometric Identification System Using Ordinal Measures", International Journal of Information Technology and Computer Science(IJITCS), vol.5, no.12, pp.86-96, 2013. DOI: 10.5815/ijitcs.2013.12.11 